\title{
Canopy Homolog 2 Expression Predicts Poor Prognosis in Hepatocellular Carcinoma with Tumor Hemorrhage
}

\author{
Dong Wang $^{\mathrm{a}}$ Zhi-Ming Wang ${ }^{\mathrm{a}}$ Sai Zhang ${ }^{\mathrm{b}} \quad$ Hai-Jun Wu $\mathrm{Wu}^{\mathrm{c}} \quad \mathrm{Yi}_{-M i n g} \mathrm{TaO}^{\mathrm{a}}$ \\ aDepartment of General Surgery, Xiangya Hospital, Central South University, Changsha, Hunan, \\ 'Institute of Medical Sciences, Xiangya Hospital, Central South University, Changsha, Hunan, \\ 'Department of Oncology, Xiangya Hospital, Central South University, Changsha, Hunan, China
}

\section{Key Words}

Hepatocellular carcinoma • Canopy homolog $2 \cdot$ Tumor hemorrhage $・$ Metastasis

\begin{abstract}
Background/Aims: Canopy homolog 2 (CNPY2) is a signature gene highly associated with tumor progression, including hepatocellular carcinoma (HCC). The presence of tumor hemorrhage $(\mathrm{TH})$ implies a fast-growing and worse tumor microenvironment. We examined a possible association between CNPY2 levels and TH and evaluated their prognostic values in patients with HCC. Methods: CNPY2 mRNA and protein levels were respectively determined in two independent cohorts of HCC specimens using quantitative real-time polymerase chain reaction (qRT-PCR) and immunohistochemistry of tissue microarrays. Kaplan-Meier survival and Cox regression analyses were executed to evaluate the prognosis of HCC. CNPY2 knockout $\mathrm{HCC}$ cell lines were established by the CRISPR/Cas9 gene editing system, and the functional role of CNPY2 in HCC cell proliferation and growth was examined in vitro and in vivo. Results: qRT-PCR showed that CNPY2 expression was significantly higher in HCC tumor tissue than in adjacent non-tumor tissue. Immunohistochemistry of HCC tissue microarrays demonstrated that CNPY2 expression was significantly correlated with TH and clinicopathological features indicating worse HCC progression. The prognostic value of CNPY2 expression and TH was validated by Cox proportional hazards analyses. Furthermore, CNPY2 knockout resulted in the significant suppression of $\mathrm{MHCC} 97 \mathrm{H}$ cell proliferation, tumor growth, and hemorrhage. Bioinformatics analysis revealed that CNPY2 was closely associated with the expression levels of 6 positive impact genes in HCC, namely, ROMO1, BOLA2, HSF1, ATG4B, ATF4, and DENR, which are implicated in the regulation of the tumor microenvironment. Conclusion: CNPY2 is an oncogene that plays a critical role in the progression of HCC with TH. CNPY2 could be exploited as a novel prognostic marker and potential target for therapeutic intervention in HCC.

D. Wang and Z.-M. Wang contributed equally to this work. 


\section{Cellular Physiology Cell Physiol Biochem 2018;50:2017-2028 \begin{tabular}{ll|l} 
and Biochemistry Published online: 10 November 2018 & $\begin{array}{l}\text { (c) } 2018 \text { The Author(s). Published by S. Karger AG, Basel } \\
\text { www.karger.com/cpb }\end{array}$
\end{tabular}}

\section{Introduction}

Hepatocellular carcinoma (HCC) constitutes a major public health problem, with 782, 000 newly diagnosed cases and 746, 000 deaths in 2012, and HCC is ranked as the third leading cause of cancer-related deaths worldwide [1]. HCC is a highly aggressive malignancy with tumor heterogeneity. The tumor microenvironment plays a pivotal role in regulating the progression of HCC [2]. Increasing evidence has revealed the high heterogeneity of the tumor microenvironment among different individuals with HCC. Change of the tumor microenvironment can result in totally different aggressive types of HCC $[3,4]$.

As an underlying key factor of the tumor microenvironment, tumor hemorrhage $(\mathrm{TH})$ is recognized to be involved in tumor growth and metastasis. It is known that during TH, red blood cells and platelets aggregate around tumor cells, facilitating the formation of cancer cell nests and providing protection against immune responses and shear stress [5-7]. TH has been demonstrated to be a poor prognostic factor for patients with HCC [8]. Therefore, exploration of the molecular contexts associated with these pathogenic changes in HCC could greatly benefit the efficacy of clinical treatments of HCC.

Canopy homolog 2 (CNPY2) is a secreted protein belonging to the canopy homolog protein family that also contains CNPY1, CNPY3, and CNPY4. Previous studies revealed that CNPY2 functions as a key pro-angiogenic protein [9]. CNPY2 was also reported to be able to promote potent tumor growth and metastasis $[10,11]$. In a gene expression profile survey, a significant increase of CNPY2 expression was linked to metastasis in primary HCC samples [12]. Although its expression was reported to be elevated during tumorigenesis, CNPY2 has not been studied extensively in HCC.

In the present study, for the first time, we investigate both CNPY2 expression and TH in patients with HCC and explore their potential correlation with clinicopathologic parameters as well as clinical outcomes. Furthermore, we knocked out CNPY2 in HCC cell lines using the CRISPR/Cas9 system, which inhibited HCC cell proliferation and attenuated growth and intratumor hemorrhage. Thus, targeting CNPY2 and TH may be a novel approach for the treatment of HCC.

\section{Materials and Methods}

\section{Patient populations and follow-up}

Two independent cohorts of subjects with HCC were enrolled in this study (training cohort: 124 HCC patients; validation cohort: 216 HCC patients). TH was reviewed by two independent pathologists in our hospital. The following procedures from our previous study were adopted [13]. Patient follow-up was terminated on January 31, 2017. All research protocols complied strictly with the REMARK guidelines for reporting prognostic biomarkers in cancer [14].

Quantitative real-time polymerase chain reaction in clinical HCC specimens

SYBRgreenfluorescent-based quantitativereal-timepolymerasechainreaction(qRT-PCR)wasperformed as described previously [15], and actin was used as a control for CNPY gene detection. The oligonucleotide sequences for the CNPY and $\beta$-actin primers were as follows: CNPY1, $5^{\prime}$-CACGGGCTATGGCATCCTG, 3'-ACTTCGATTAACCGCAAGT; CNPY2, 5'-ACAAGGCATCCGAATCGACTC, $3^{\prime}$-GTCAGCCTCTCGGGAAAAGAA; CNPY3, 5'-CGGAGCTGAGGAGAACGAC, 3'-ATAGCCCGTGCCAATCACC; CNPY4, 5' -GTGCCTTACAGCGTTTCAGAG, 3'-ACCCTTGGCATATCTCAGTGA; and $\beta$-actin, 5' -GGACTTCGAGCAAGAGATGG, 3'-AGCACTGTGTTGGCGTACAG.

Tissue microarray and immunohistochemistry analyses

A tissue microarray containing 340 pairs of HCC and adjacent non-tumor (NT) tissues was constructed. Immunohistochemistry was performed as described previously [16]. An anti-CNPY2 polyclonal antibody (Abcam, Cambridge, MA, Cat\# ab233136) diluted at 1:1000 was used as the primary antibody. Peroxidase-

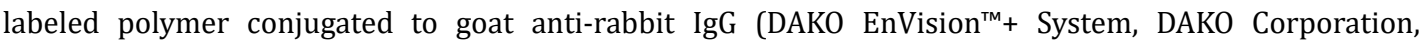
Carpinteria, CA, Cat\# K5007) was applied for $30 \mathrm{~min}$ at room temperature and color was developed using a 


\section{Cellular Physiology Cell Physiol Biochem 2018;50:2017-2028

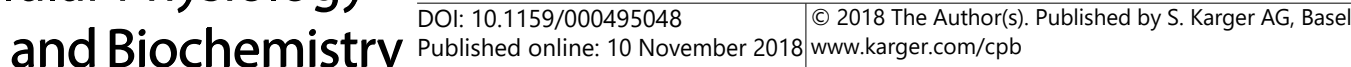 \\ Wang et al.: Role of Novel Metastasis-Related Gene CNPY2 in HCC}

3, 3'-diaminobenzidine solution. CNPY2 expression was assessed as cytoplasmic or membranous staining. Staining was assessed using the H-score method [17].

\section{HCC cell lines}

MHCC97H and HCCLM3 cell lines were purchased from the Liver Cancer Institute of Fudan University (Shanghai, China). HepG2, Bel7404, SMCC7721, Huh7, Hep3B, and L02 cells were purchased from the American Type Culture Collection (Rockville, MD). The cells were cultured as described previously [15].

\section{Establishment of CNPY2 knockout HCC cell lines}

CNPY2-deficient HCC cells were established using the CRISPR/Cas9 system. The guide RNA was selected with the assistance of the CRISPR design tool according to the standard protocol [18]. Briefly, the sgRNA sequences ofCNPY2 were 5'-CAAGAACTACGTACGTGTAG-3'; bottom strand:5'-TCTTTCCGGATCAATCCAGA-3'. For control cells, no guide RNA was inserted. Each experimental replicate was performed using the HCC cell line MHCC97H.

\section{Western blotting}

Western blot analyses were performed as described previously [19]. Antibody dilutions were 1:1000 for the anti-CNPY2 polyclonal antibody (Abcam, Cat\# ab233136) and 1:5000 for the anti- $\beta$-actin mouse monoclonal antibody (Sigma-Aldrich, St. Louis, MO, Cat\# A1978).

\section{Cell proliferation assay}

A cell proliferation assay was performed using a Cell Counting Kit-8 according to the manufacturer's protocol (MedchemExpress, Monmouth Junction, NJ, Cat\# HY-K0301). The cells (800 cells/well) were seeded in 96-well plates and incubated for different periods of time. The cells were treated and stained. Fluorescence was measured at each time point. Each experiment was repeated three times.

\section{Clonogenic formation assay}

The cells were seeded in 6-well plates (1000 cells/well). After incubation for 14 days, the colonies that formed were washed with cold phosphate-buffered saline buffer, fixed with $4 \%$ paraformaldehyde for 15 min, and stained with $0.2 \%$ crystal violet (Thermo Fisher Scientific, Asheville, NC, Cat\# R40053). The colonies were quantified from three independent experiments per treatment group.

\section{Tumor xenograft model}

All animal work was conducted in accordance with protocols approved by the Animal Care and Use Committee at Central South University, Changsha, China. A subcutaneous HCC mouse model was established according to an existing protocol [15]. MHCC97H cells $\left(2.0 \times 10^{6}\right.$ cells/mouse) stably transfected with vectors were injected subcutaneously into the right axilla $(200 \mu \mathrm{L})$. All nude mice were killed at 35 days after tumor implantation.

\section{Bioinformatics analysis}

Data mining was performed using a previously established microarray database (GEO Submission: GSE40367) [12]. Gene expression profiling interactive analysis (GEPIA) was used to compare the expression profiles of CNPY genes [20]. The Tumor Immune Estimation Resource (TIMER) web server was used to study the correlation of CNPY2 mRNA expression and a pair of user-defined genes in liver hepatocellular carcinoma (LIHC) [20].

\section{Statistical analysis}

Quantitative values are presented as the mean \pm standard deviation (SD) or median (range). Paired $t$ and Student's tests were used for paired and unpaired continuous data, respectively. Comparisons between different groups were made using Student's $t$ test or analysis of variance as appropriate in the in vitro study. Kaplan-Meier curves were analyzed by the log-rank test. A Cox proportional hazards regression model was used to determine if CNPY2 expression was an independent prognostic indicator. $P<0.05$ was considered to be statistically significant. All statistical analyses were performed using GraphPad Prism software (GraphPad Software, San Diego, CA). 


\section{Results}

CNPY2 is frequently up-regulated in HCC

To assess the potential effect of canopy family members on HCC, we first determined the relative transcription levels of known CNPY genes, CNPY1-4, in 25 pairs of human HCC specimens by qRT-PCR. The data showed that both CNPY2 and CNPY4 mRNA expression was elevated in HCC tissues compared with their corresponding NT tissues, whereas the expression of the other canopy family members was not altered significantly (Fig. 1A). Intriguingly, survival analysis from the GEPIA web server demonstrated that only the mRNA level of CNPY2 was indicative of a poorer survival rate, both for overall survival (OS; $P=$ 0.013, Fig. 1B) and disease-free survival (DFS; $P=0.00055$, Fig. 1C); therefore, we focused on CNPY2 for further analyses.

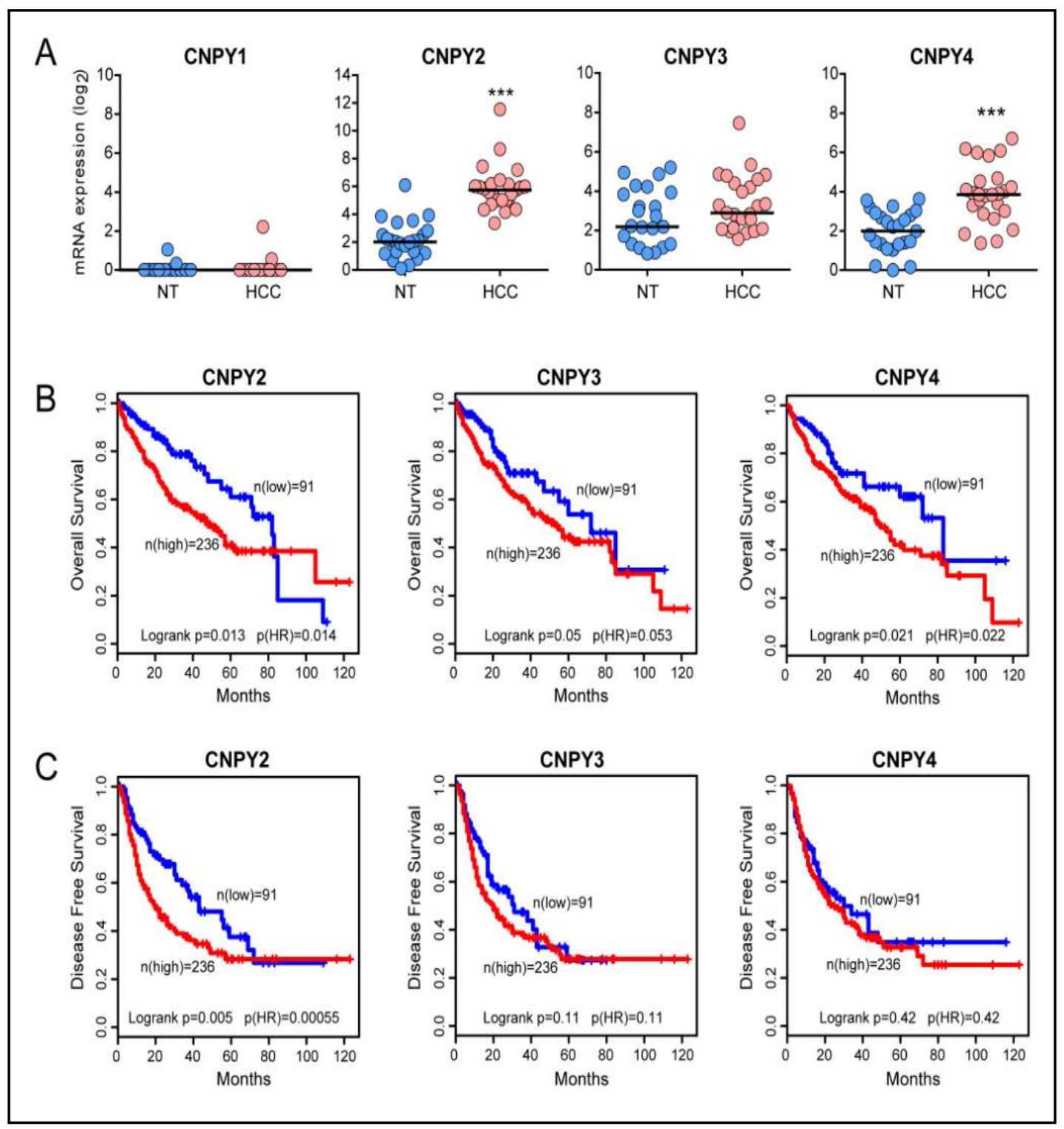

Fig. 1. CNPY2 is strongly expressed in HCC. (A) Transcript levels of the canopy family members in an initial cohort of 25 pairs of human HCC and the corresponding NT liver tissues. The OS (B) and DFS rates (C) were compared between the CNPY2 $2^{\text {high }}$ and CNPY $2^{\text {low }}$ groups. ${ }^{* *} \mathrm{P}<0.001$, unless otherwise indicated. 


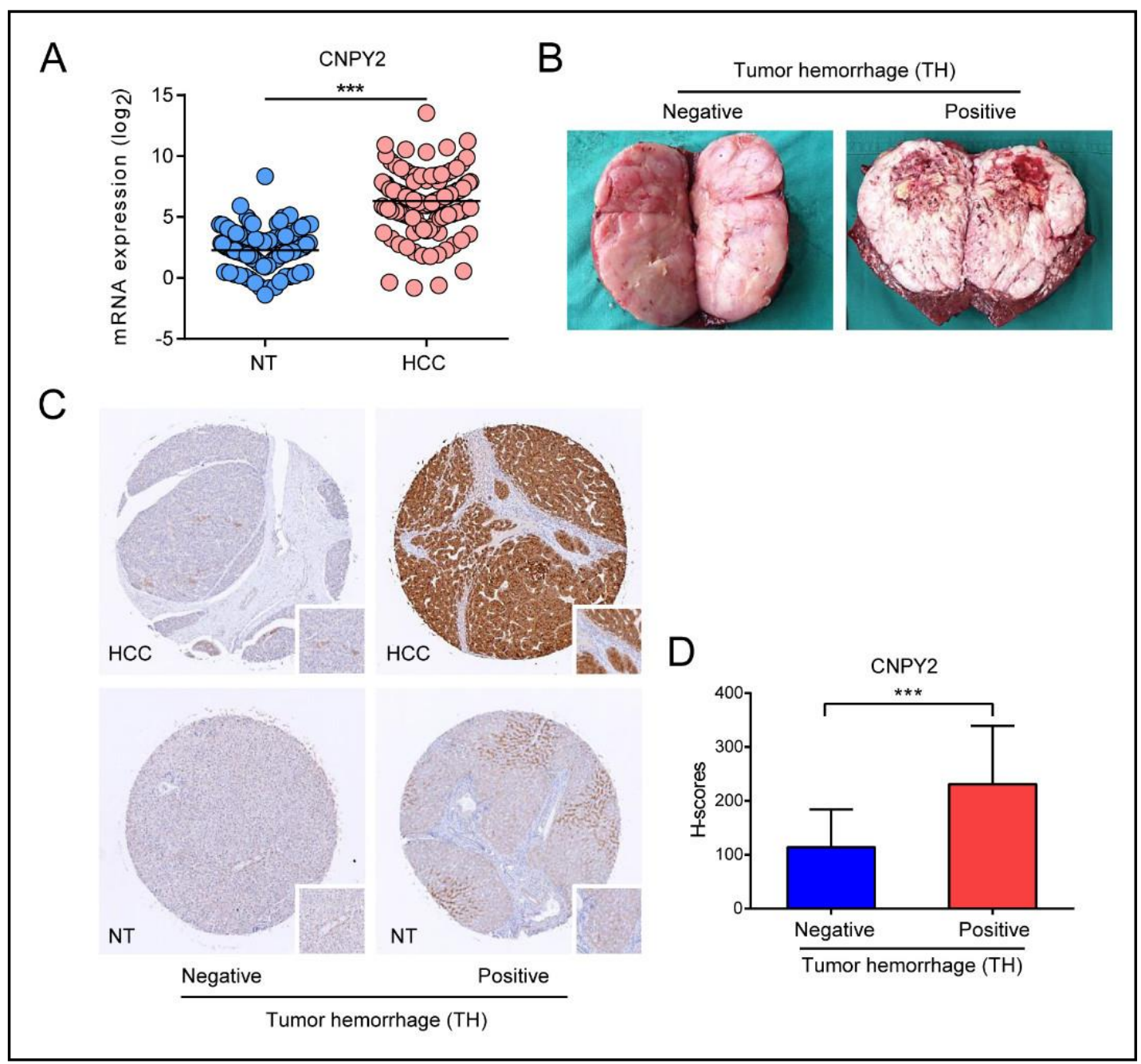

Fig. 2. Increased expression of CNPY2 is related to a high risk of TH. (A) Validation of the mRNA expression levels of CNPY2 in an expanded cohort of 124 cases of paired human HCC and corresponding NT tissues. (B) Representative gross specimens of HCC with/without TH. (C) Representative immunohistochemical staining characteristics of CNPY2 expression in NT, TH-negative tumor, and TH-positive tumor. (D) CNPY2 immunohistochemistry staining score analysis showed that CNPY2 expression was much higher in patients with TH of HCC than in those without TH.

\section{Association between CNPY2 and TH}

Conventional qRT-PCR was performed in an expanded training cohort of 124 pairs of HCC and NT samples; CNPY2 mRNA was up-regulated in tumors compared with NT (6.184 \pm 0.215 versus $2.371 \pm 0.135$, respectively, $P<0.001$, Fig. 2 A). The overexpression of CNPY2 mRNA (defined as a $>2.0$-fold increase) was detected in 68/124 (54.8\%) of HCC samples. There was a significant association between the overexpression of CNPY2 mRNA and the presence of satellite nodules, venous invasion, $\mathrm{TH}$, tumor size, and tumor, node, metastasis (TNM) stage (Table 1). To confirm this result, 124 HCC tumors were classified into subgroups by the presence or absence of TH (Fig. 2B); we examined CNPY2 protein expression in each cohort by immunohistochemistry (Fig. 2C). As expected, a consistent result was obtained; that is, tumors with TH showed higher CNPY2 expression compared with tumors without TH (Fig. 2D). 
Association between CNPY2 and poor survival

Cox multivariate analysis indicated that CNPY2 protein expression was an independent positive prognostic factor in the training cohort (hazard ratio $=1.932,95 \%$ confidence interval 1.329-4.524; Table 2). The CNPY2 ${ }^{\text {high }}$ expression group exhibited worse OS and shorter recurrence-free survival (RFS) than those in the CNPY2 $2^{\text {low }}$ expression group (Fig. 3A). The 1-, 3-, and 5-year OS rates in the high expression group were significantly lower than those in the low expression group (65.9\% versus $82.1 \%, 31.8 \%$ versus $61.6 \%$, and $17.3 \%$ versus $44.9 \%$, respectively). The 1-, 3-, and 5-year RFS rates were markedly lower in the high expression group compared with the low expression group (44.1\% versus $62.5 \%, 19.4 \%$ versus $35.3 \%$, and $19.4 \%$ versus $25.4 \%$, respectively). Moreover, these findings were confirmed in another validation cohort containing 216 HCC patients (Fig. 3B). Taken together, the level of CNPY2 expression may be a clinical predictor of HCC prognosis.

\section{CNPY2 knockout suppresses HCC cell proliferation}

Consistent with the results from the tissues, western blot analysis showed that the protein expression levels of CNPY2 were significantly increased in seven established HCC cell lines in comparison with the nontransformed hepatic cell line L02 (Fig. 4A). To investigate the function of CNPY2 in HCC, we established CNPY2 knockout (KO) in MHCC97H cell lines using CRISPR/Cas 9 technology. Cell proliferation assays demonstrated that CNPY2 KO potently slowed cell proliferation compared with wild-type (WT) cells, and evaluated the effects of CNPY2 in these cells (Fig. 4B). The suppressive rates in the CNPY2-deficient colonies derived from MHCC97H cells were $37.5 \%$ (KO-3) and 33.3\% (KO-5) compared with WT cells at day 7. Cell colony assays further identified similar suppressive effects of CNPY2 KO (Fig. 4C). CNPY2 KO significantly suppressed the colony forming ability of MHCC97H cells, with the inhibitory

Table 1. Correlation between CNPY2 gene expression and clinicopathological characteristics in patients with HCC ( $\mathrm{n}=$ 124). AFP, $\alpha$-fetoprotein; TNM, tumor, node, metastasis; data are expressed as counts or mean (range). *Student's t test. "Fisher's exact test or chi-square test

\begin{tabular}{|c|c|c|c|c|}
\hline Clinicopathologic variable & Parameter & $\begin{array}{l}\text { High expression } \\
\qquad(\mathrm{n}=68)\end{array}$ & $\begin{array}{l}\text { Low expression } \\
\quad(\mathrm{n}=56)\end{array}$ & P-value \\
\hline Age, years & & $50.4(18.5-76.5)$ & $47.5(19.1-73.2)$ & $0.106^{*}$ \\
\hline Serum albumin, g/mL & & $39.5(30.2-49.5)$ & $41.3(31.0-48.7)$ & $0.532^{*}$ \\
\hline Tumor size, $\mathrm{cm}$ & & $10.8(4.8-16.5)$ & $7.8(5.0-15.7)$ & $0.015^{*}$ \\
\hline \multirow[t]{2}{*}{ Sex } & Male & 55 & 42 & $0.430^{\#}$ \\
\hline & Female & 13 & 14 & \\
\hline \multirow[t]{2}{*}{$\mathrm{AFP}, \mathrm{ng} / \mathrm{mL}$} & $\leq 20$ & 28 & 22 & $0.831^{\#}$ \\
\hline & $>20$ & 40 & 34 & \\
\hline \multirow[t]{2}{*}{ Liver cirrhosis } & No & 7 & 3 & $0.315^{\#}$ \\
\hline & Yes & 61 & 53 & \\
\hline \multirow[t]{2}{*}{ Tumor encapsulation } & No & 42 & 40 & $0.258^{\#}$ \\
\hline & Complete & 26 & 16 & \\
\hline \multirow[t]{2}{*}{ Tumor number } & Single & 30 & 34 & $0.066^{\#}$ \\
\hline & Multiple & 38 & 22 & \\
\hline \multirow[t]{2}{*}{ Venous invasion } & No & 17 & 27 & 0.007 \\
\hline & Yes & 51 & 29 & \\
\hline \multirow[t]{2}{*}{ Satellite nodules } & No & 23 & 31 & 0.016 \\
\hline & Yes & 45 & 25 & \\
\hline \multirow[t]{2}{*}{ Edmondson grade } & I-II & 37 & 20 & 0.188 \\
\hline & III-IV & 31 & 36 & \\
\hline \multirow[t]{2}{*}{ Tumor hemorrhage } & No & 14 & 24 & 0.007 \\
\hline & Yes & 54 & 32 & \\
\hline \multirow[t]{2}{*}{ TNM stage } & I-II & 26 & 34 & 0.013 \\
\hline & III & 42 & 22 & \\
\hline
\end{tabular}

Table 2. Univariate and multivariate analyses of prognostic factors with RFS and OS in patients with HCC $(\mathrm{n}=124)$ HCC, hepatocellular carcinoma; RFS, recurrence-free survival; OS, overall survival; HR, hazard ratio; CI, confidence interval; NA, not adopted; AFP, $\alpha$-fetoprotein; TH, tumor hemorrhage; TNM, tumor, node, metastasis. Significant differences are shown in bold. †Cox proportional hazards regression

\begin{tabular}{|c|c|c|c|c|}
\hline Clinicopathologic variable & $\begin{array}{c}\text { RFS } \\
\text { HR }(95 \% \mathrm{CI})\end{array}$ & P-value & $\begin{array}{c}\text { OS } \\
\text { HR }(95 \% \mathrm{CI})\end{array}$ & P-value \\
\hline \multicolumn{5}{|l|}{ Univariate analysis $\dagger$} \\
\hline Sex (male vs. female) & $1.201(0.899-1.562)$ & 0.116 & $1.264(0.876-1.571)$ & 0.167 \\
\hline Age, years (>60 vs. $\leq 60$ ) & $1.078(0.654-1.215)$ & 0.352 & $1.178(0.924-1.542)$ & 0.106 \\
\hline Serum albumin, $\mathrm{g} / \mathrm{L}(\leq 35$ vs. $>35)$ & $1.206(0.944-2.218)$ & 0.075 & $1.224(0.952-1.421)$ & 0.092 \\
\hline Liver cirrhosis (presence vs. absence) & $1.138(1.016-2.564)$ & 0.042 & $1.428(1.105-2.287)$ & 0.033 \\
\hline AFP, ng/mL (>20 vs. $\leq 20)$ & $1.536(1.132-2.567)$ & 0.013 & $1.435(1.105-2.762)$ & 0.027 \\
\hline Tumor size, $\mathrm{cm}(>5$ vs. $\leq 5)$ & $1.475(1.284-3.146)$ & 0.024 & $1.654(1.208-2.842)$ & 0.010 \\
\hline Tumor number (multiple vs. single) & $1.518(1.102-3.477)$ & 0.008 & $1.504(1.123-2.647)$ & 0.021 \\
\hline Tumor encapsulation (none vs. complete) & $1.195(0.972-1.463)$ & 0.067 & $1.478(1.098-2.321)$ & 0.027 \\
\hline Venous invasion (presence vs. absence) & $2.167(1.349-3.854)$ & 0.002 & $2.236(1.467-3.965)$ & $<0.0001$ \\
\hline Edmondson grade (III/IV vs. I/II) & $1.418(1.072-2.1$ & 0.024 & $1.214(0.935-1.624)$ & 0.084 \\
\hline Satellite nodules (presence vs. absence) & $1.456(1.113-2.326)$ & 0.012 & $1.428(1.105-2.287)$ & 0.033 \\
\hline TH (presence vs. absence) & $1.742(1.365-4.533)$ & 0.005 & $1.746(1.285-4.068)$ & 0.012 \\
\hline TNM s & $1.412(1.054-1.938)$ & 0.030 & $1.678(1.239-3.642)$ & 0.019 \\
\hline CNPY2 expression level (high vs. low) & $1.945(1.231-4.526)$ & 0.002 & $2.058(1.453-5.462)$ & 0.001 \\
\hline \multicolumn{5}{|l|}{ Multivariate analysis $\dagger$} \\
\hline & NA & & NA & \\
\hline Age, years ( $>60$ vs. $\leq 60)$ & $\mathrm{NA}$ & & NA & \\
\hline lbumin, g/L ( $\leq 35$ vs. $>35$ ) & NA & & NA & \\
\hline Liver & $1.097(0.738-1.546)$ & NS & $1.096(0.832-1.405)$ & NS \\
\hline AFP, $n$ & $1.206(0.944-2.2)$ & NS & $1.125(0.796-1.954)$ & NS \\
\hline Tumor & $1.079(0.833-1.364)$ & NS & $1.096(0.832-1.405)$ & NS \\
\hline Tumor number (multiple v & $1.472(1.071-2.488)$ & 0.029 & $1.514(1.182-2.937)$ & 0.012 \\
\hline Tumor encapsulation (non & NA & & $1.086(0.923-1.687)$ & NS \\
\hline Venous invasion (presence vs. absence) & $1.921(1.354-4.275)$ & 0.006 & $1.864(1.264-4.946)$ & 0.008 \\
\hline Edmon & $1.248(0.864-1.612)$ & NS & NA & \\
\hline e nodules (presence vs. absence) & $1.263(1.024-1.682)$ & 0.038 & $1.092(1.023-1.756)$ & 0.042 \\
\hline TH (presence vs. absence) & $1.462(1.142-2.843)$ & 0.021 & $1.867(1.216-4.235)$ & 0.004 \\
\hline TNM stage (II/III vs. I) & $1.523(1.179-2.164)$ & 0.012 & $1.537(1.205-2.864)$ & 0.010 \\
\hline CNPY2 expression level (high vs. low) & $2.167(1.349-3.854)$ & $<0.0001$ & $1.932(1.329-4.524)$ & 0.003 \\
\hline
\end{tabular}


Fig. 3. High expression of CNPY2 protein indicates a worse clinical outcome for patients with HCC. (A and B) Kaplan-Meier curves depicting OS and RFS according to the expression levels of CNPY2 in the training cohort $(n=124)$ and in the validation cohort $(\mathrm{n}=216)$. The median value of $\mathrm{H}$-scores was used as the cut-off for low and high CNPY2 expression. The P-values were calculated using the log-rank test.

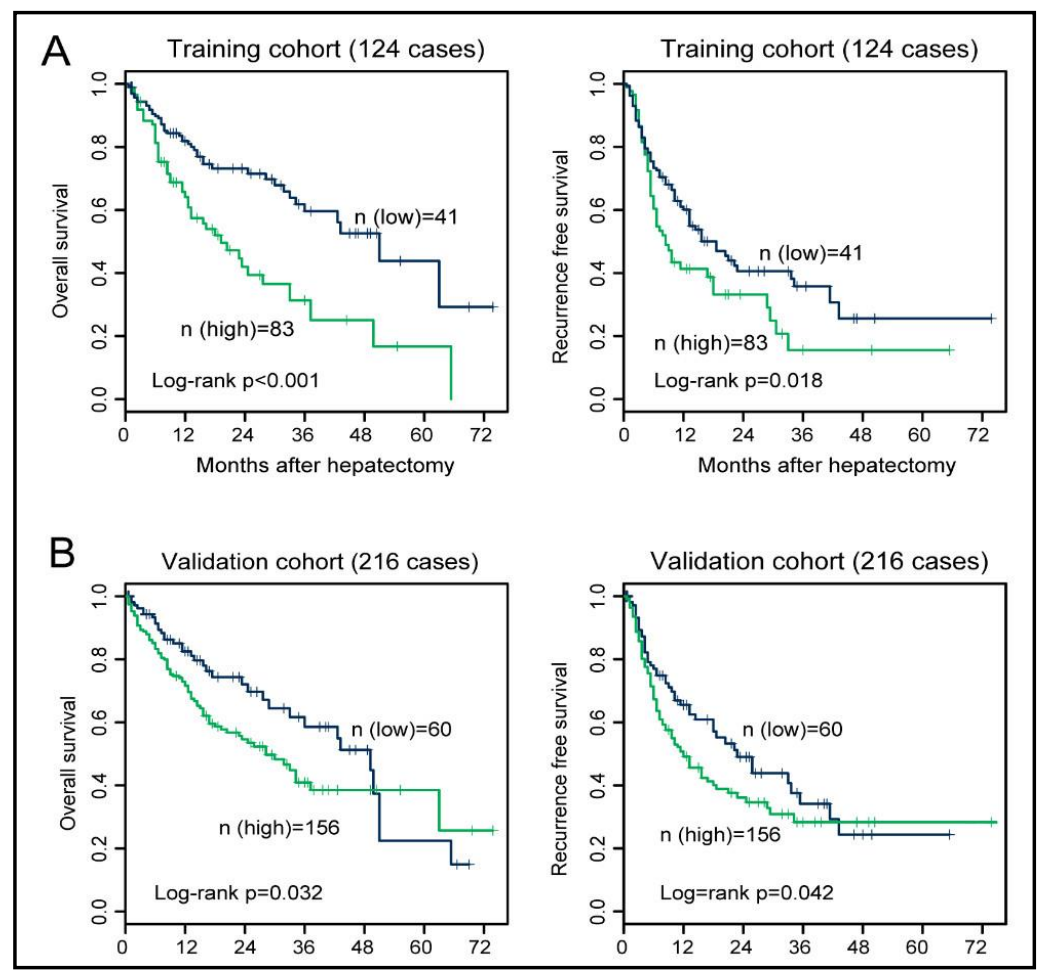

Fig. 4. CNPY2 KO reduces the proliferation of HCC cells in vitro. (A) Western blot analysis confirmed the expression of CNPY2 in HCC cells. (B) CNPY2 KO by CRISPR/Cas9 technology in MHCC97H cells was confirmed by western blot analysis. CNPY2 KO slows cell proliferation. (C) A colony formation assay was performed in CNPY2-deficient colonies derived from MHCC97H cells and WT cells. The values are expressed as the mean \pm SD of three independent experiments.

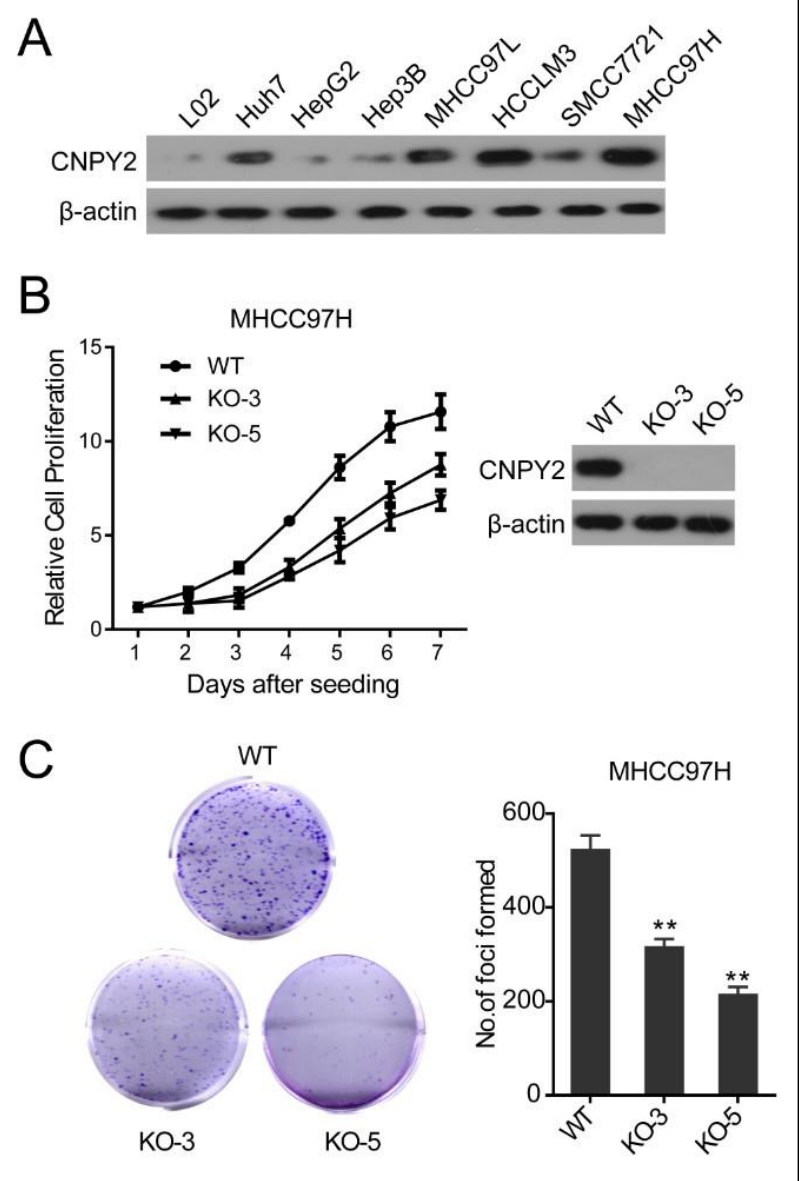




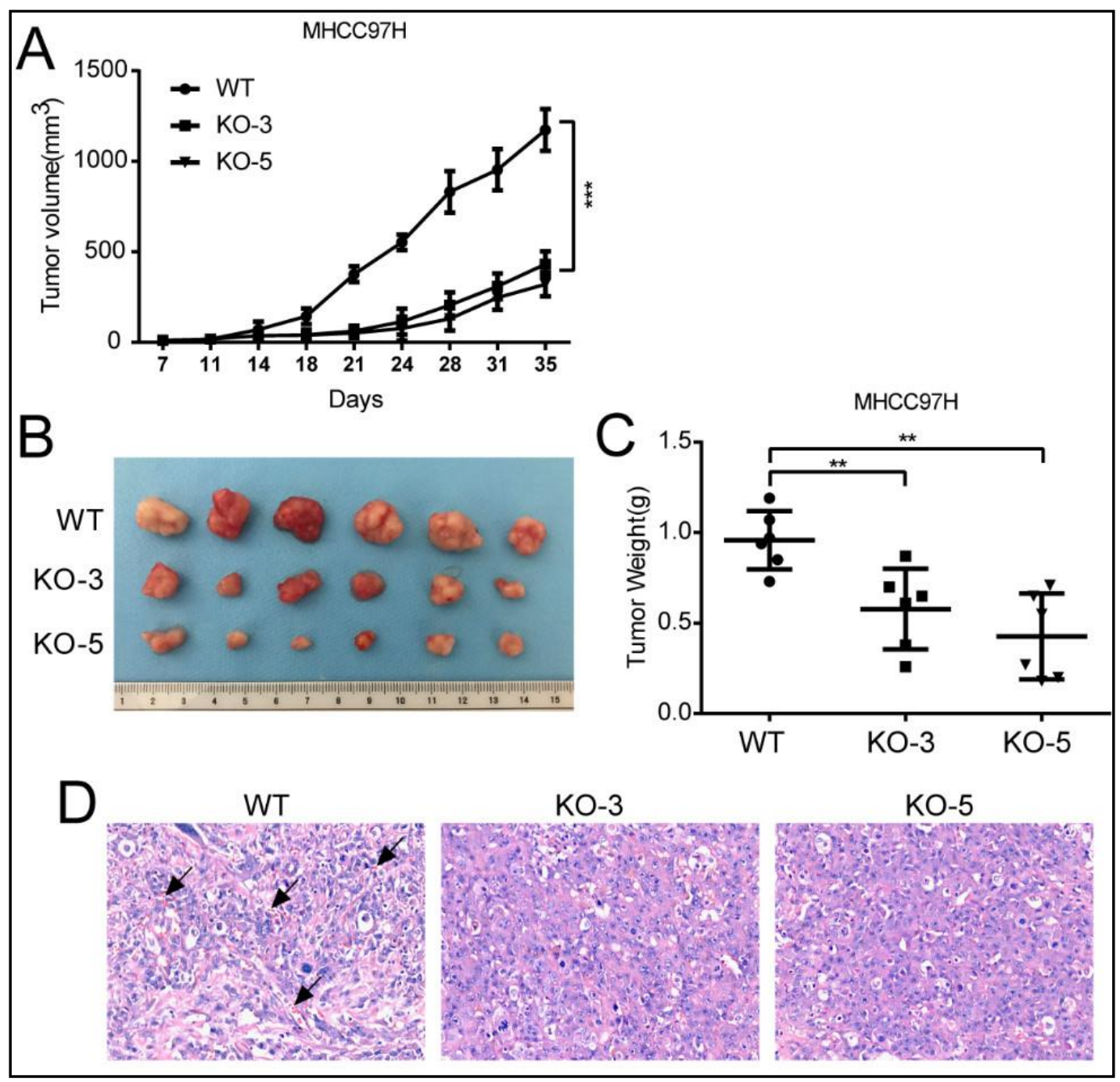

Fig. 5. CNPY2 KO decreases the tumorigenicity of HCC cells in a mouse xenograft model. (A) Tumor growth curves in the three groups are shown at the indicated days after injection of MHCC97H cells (WT, KO-3, and KO-5). Xenograft tumor volumes of each group were measured twice a week. (B) Representative images of subcutaneous tumors in nude mice injected with the indicated cells. (C) Final tumor weights are summarized in the dot chart. Average tumor weight is expressed as the mean \pm SD of 6 mice. (D) Hematoxylin and eosin staining demonstrated that CNPY2 KO inhibited the TH phenotype of HCC in vivo (black arrow indication).

rates being 45.3\% and 33.6\% in MHCC97H KO-3 and KO-5 cells, respectively. These results suggest an oncogenic role for CNPY2 in HCC tumor progression.

\section{CNPY2 KO suppresses tumor growth and TH}

As described above, CNPY2 KO could suppress MHCC97H cell proliferation in vitro. Here, we attempted to determine whether CNPY2 KO had the same suppressive effects on HCC tumor growth in vivo. Subcutaneous xenograft experiments were applied to investigate the in vivo tumorigenicity of CNPY2 further. Compared with the WT group, we found that CNPY2 KO strongly suppressed tumor growth (Fig. 5A). At day 35, all tumors were removed (Fig. 5B) and weighed (Fig. 5C). The average tumor weights in the $\mathrm{KO}-5, \mathrm{KO}-3$, and WT groups were $0.426 \mathrm{~g}, 0.578 \mathrm{~g}$, and $0.958 \mathrm{~g}$, respectively. Statistical analysis showed significant suppression of tumor growth by CNPY2 KO. Importantly, we found that TH was significantly decreased in xenografts derived from MHCC97H-KO-CNPY2 cells (Fig. 5D).

\section{KARGER}




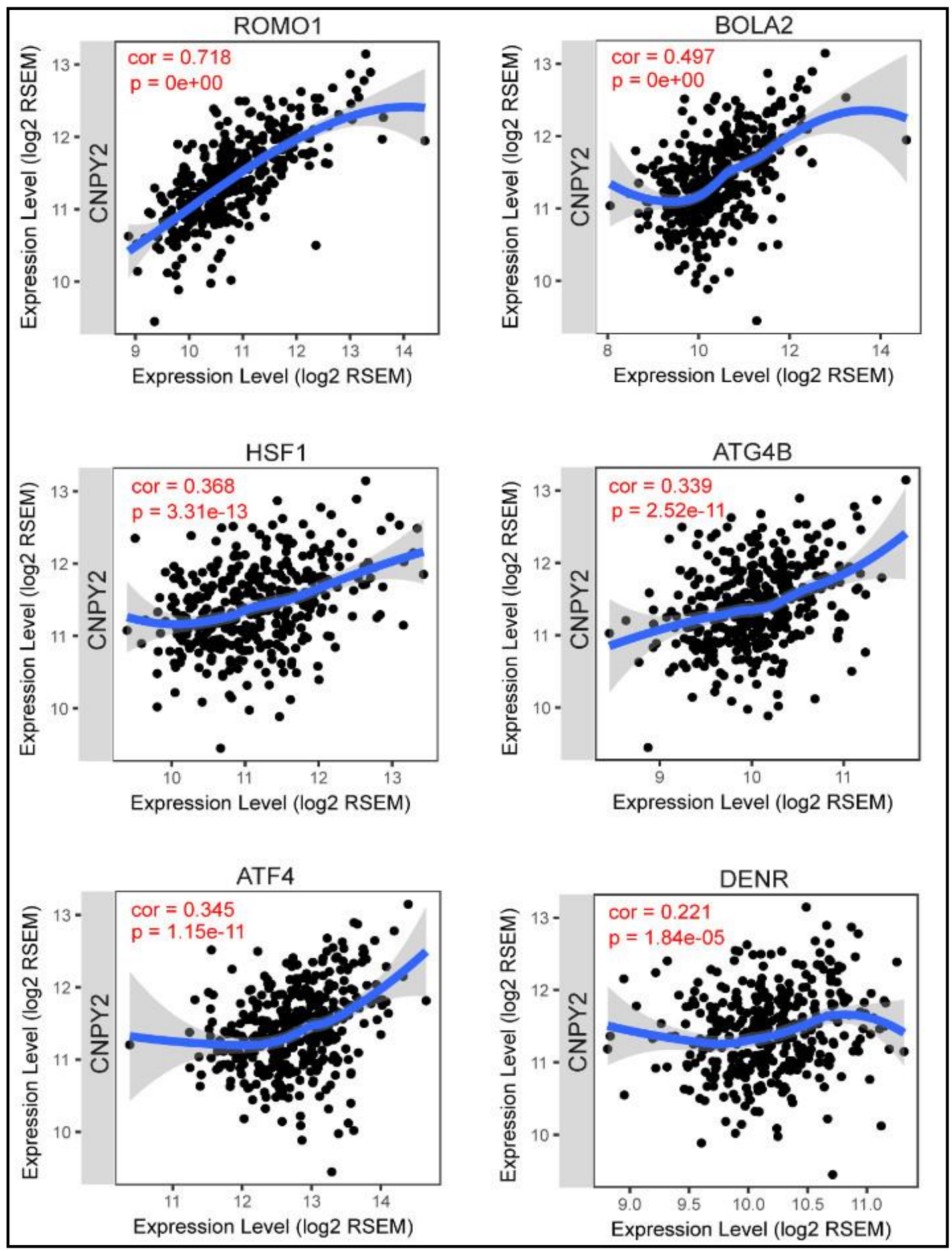

Fig. 6. Spearman's correlation analysis of the tumor markers ROM01, BOLA2, HSF1, ATG4B, ATF4, and DENR with CNPY2 expression in the HCC profiles of The Cancer Genome Atlas dataset.

CNPY2 expression is associated with the molecular context of the tumor microenvironment As shown in Fig. 6, by using the TIMER web server, a positive correlation was observed between CNPY2 mRNA expression and defined genes in LIHC, such as reactive oxygen species modulator 1 (ROMO1) [21], bolA family member 2 (BOLA2) [22], heat shock transcription factor 1 (HSF1), autophagy related 4B (ATG4B) [23], activating transcription factor 4 (ATF4) [24], and density-regulated protein (DENR) [25], which are suggested to have roles

\section{KARGER}




\section{Cellular Physiology Cell Physiol Biochem 2018;50:2017-2028

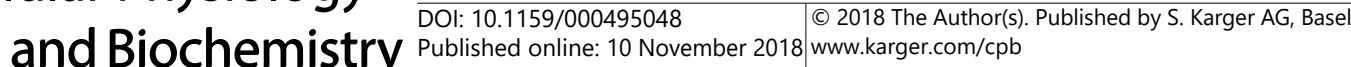

Wang et al.: Role of Novel Metastasis-Related Gene CNPY2 in HCC

in regulating tumor growth and microenvironment. TH implies a fast-growing and worse tumor immunological microenvironment [8]. Further studies of CNPY2 in HCC will be of interest to clarify its precise role in TH and cell growth.

\section{Discussion}

As one of the leading causes of cancer-related death, HCC is characterized by its rapid progression and frequent recurrence and metastasis. We previously demonstrated that $\mathrm{TH}$ was involved in the fast growth of HCC; therefore, the presence of TH in HCC specimens may be one of the key malignant clinicopathological features of HCC. Understanding the underlying molecular mechanisms in the development of TH is very important.

$\mathrm{TH}$ is a common phenomenon in solid tumors, such as liver cancer and colorectal cancer. In most situations, the hemorrhage is caused by ruptured blood vessels or tumor invasion, and red blood cells spill out of the vessels. TH mainly includes red blood cells, white blood cells, and platelets, and the components of the hemorrhage have been demonstrated to promote cancer progression. Platelets can induce angiogenesis in the tumor by promoting epithelial-mesenchymal transition, while red and white blood cells can remodel the tumor stroma and change the inflammatory microenvironment, which can effectively promote tumor growth.

Members of the canopy family of proteins are characterized by an endoplasmic reticulum retention signal at the $\mathrm{C}$ terminus and have been implicated in human cancer [26]. However, the prognostic significance of CNPY2 expression in HCC is still unclear. In the present study, we found that the level of CNPY2 overexpression was positively correlated with the presence of satellite nodules, venous invasion, TH, and TNM stage. Moreover, Kaplan-Meier survival analysis showed that HCC patients with CNPY2 ${ }^{\text {high }}$ expression had shorter OS than those with CNPY2 ${ }^{\text {low }}$ expression. We hypothesized that CNPY2 might act as an oncogene to enhance the development of HCC. In order to confirm this, our loss-of-function assays showed that the loss of CNPY2 reduced HCC cell proliferation in vitro and decreased tumor growth and $\mathrm{TH}$ in vivo. These results suggested that CNPY2 may act as a critical tumor promoter in the progression of HCC, which leads to a poor clinical outcome in patients with HCC.

The precise mechanism underlying this CNPY2-mediated TH in tumor growth remains to be determined. In this work, we performed TIMER analysis, and the results indicated that CNPY2 was highly associated with the tumor immunological microenvironment. The tumor microenvironment consists of a number of components including growth factors and inflammatory cytokines, stromal cells, extracellular matrix proteins, and several different cell types. The tumor microenvironment induces endoplasmic reticulum stress in tumor cells, an event that can promote progression. Moreover, CNPY2 was recently identified as an initiator of the unfolded protein response (UPR) in liver inflammation and regenerative proliferation [27]. Apoptosis occurs by distinct pathways that involve cell autophagy or the endoplasmic reticulum [28]. In this role, UPR-associated HCC progression consistently exhibits more inflammation, epithelial-mesenchymal transition, venous invasion, and a poorer prognosis [29]. These findings would contribute to the growing knowledge base on the mechanism of UPR-induced hepatocarcinogenesis.

The occurrence and progression of HCC are linked tightly to progressive hepatic metabolic syndrome associated with chronic inflammation [30]. HSF1, a major transactivator of stress proteins, increases survival by protecting cells against environmental stressors [31]. DENR is a key role in supporting proliferation and tissue growth. We found that CNPY2 overexpression was significantly associated with HSF1 and DENR. Studies performed on human HCC patients with metastasis showed a significant increase of CNPY2 protein levels and activity, arguing for a functional link between GSNOR-dependent $S$-nitrosylation and HCC existing also in humans [12]. Chronic oxidative stress produced by mitochondria promotes hepatocarcinogenesis and tumor progression [32]. Thus, we could speculate that CNPY2 might promote the development of HCC and maintain cancer cell growth under metabolic 


\section{Cellular Physiology Cell Physiol Biochem 2018;50:2017-2028

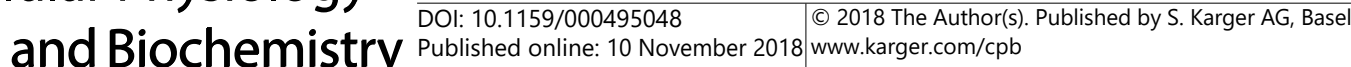

Wang et al.: Role of Novel Metastasis-Related Gene CNPY2 in HCC

stress. Further studies of CNPY2 in HCC will be of interest to clarify its precise role in TH and cell growth.

\section{Conclusion}

In conclusion, our data indicated that CNPY2 is overexpressed in HCC and could serve as a key emerging molecule in HCC cell growth and TH. These findings have important implications for identifying new therapeutic targets in HCC.

\section{Acknowledgements}

We thank The Cancer Genome Atlas for their efforts and providing data. The authors would like to thank T. CH. TAO for his indispensable support. This study was supported by grants from the National Nature Science Foundation of China (No. 81372630) and from the Nature Science Foundation of Hunan Province (No. 12JJ3118).

\section{Disclosure Statement}

The authors have no conflicts of interest to declare.

\section{References}

1 Siegel RL, Miller KD, Jemal A: Cancer statistics, 2016. CA: a cancer journal for clinicians 2016;66:7-30.

2 Xue R, Li R, Guo H, Guo L, Su Z, Ni X, Qi L, Zhang T, Li Q, Zhang Z, Xie XS, Bai F, Zhang N: Variable Intra-Tumor Genomic Heterogeneity of Multiple Lesions in Patients With Hepatocellular Carcinoma. Gastroenterology 2016;150:998-1008.

3 Giannelli G, Rani B, Dituri F, Cao Y, Palasciano G: Moving towards personalised therapy in patients with hepatocellular carcinoma: the role of the microenvironment. Gut 2014;63:1668-1676.

4 Hernandez-Gea V, Toffanin S, Friedman S, Llovet J: Role of the microenvironment in the pathogenesis and treatment of hepatocellular carcinoma. Gastroenterology 2013;144:512-527.

5 Cubillos-Ruiz J, Bettigole S, Glimcher L: Tumorigenic and Immunosuppressive Effects of Endoplasmic Reticulum Stress in Cancer. cell 2017;168:692-706.

6 Yin T, He S, Liu X, Jiang W, Ye T, Lin Z, Sang Y, Su C, Wan Y, Shen G, Ma X, Yu M, Guo F, Liu Y, Li L, Hu Q, Wang Y, Wei Y: Extravascular red blood cells and hemoglobin promote tumor growth and therapeutic resistance as endogenous danger signals. J Immunol 2015;194:429-437.

7 Bihari C, Rastogi A, Shasthry SM, Bajpai M, Bhadoria AS, Rajesh S, Mukund A, Kumar A, Sarin SK: Platelets contribute to growth and metastasis in hepatocellular carcinoma. APMIS 2016;124:776-786.

8 Hu K, Wang ZM, Li JN, Zhang S, Xiao ZF, Tao YM: CLEC1B Expression and PD-L1 Expression Predict Clinical Outcome in Hepatocellular Carcinoma with Tumor Hemorrhage. Transl Oncol 2018;11:552-558.

-9 Guo J, Zhang Y, Mihic A, Li S-H, Sun Z, Shao Z, Wu J, Weisel RD, Li R-K: A secreted protein (Canopy 2, CNPY2) enhances angiogenesis and promotes smooth muscle cell migration and proliferation. Cardiovascular research 2015;105:383-393.

10 Ito S, Ueda T, Ueno A, Nakagawa H, Taniguchi H, Kayukawa N, Miki T: A genetic screen in Drosophila for regulators of human prostate cancer progression. Biochem Biophys Res Commun 2014;451:548-555.

11 Taniguchi H, Ito S, Ueda T, Morioka Y, Kayukawa N, Ueno A, Nakagawa H, Fujihara A, Ushijima S, Kanazawa M, Hongo F, Ukimura O: CNPY2 promoted the proliferation of renal cell carcinoma cells and increased the expression of TP53. Biochem Biophys Res Commun 2017;485:267-271.

12 Ye Q-H, Zhu W-W, Zhang J-B, Qin Y, Lu M, Lin G-L, Guo L, Zhang B, Lin Z-H, Roessler S: GOLM1 modulates EGFR/RTK cell-surface recycling to drive hepatocellular carcinoma metastasis. Cancer cell 2016;30:444458. 


\section{Cellular Physiology Cell Physiol Biochem 2018;50:2017-2028 \begin{tabular}{l|l|l|l|l} 
and & DOI: 2018 The Author(s). Published by S. Karger AG, Basel
\end{tabular}

13 Tao Y, Liu Z, Liu H: Dickkopf-1 (DKK1) promotes invasion and metastasis of hepatocellular carcinoma. Dig Liver Dis 2013;45:251-257.

14 Altman DG, McShane LM, Sauerbrei W, Taube SE: Reporting Recommendations for Tumor Marker Prognostic Studies (REMARK): explanation and elaboration. PLoS Med 2012;9:e1001216.

15 Tao YM, Huang JL, Zeng S, Zhang S, Fan XG, Wang ZM, Yang HX, Yuan XH, Wang P, Wu F, Luo J, Zeng DY, Shen H: BTB/POZ domain-containing protein 7: epithelial-mesenchymal transition promoter and prognostic biomarker of hepatocellular carcinoma. Hepatology 2013;57:2326-2337.

$>16$ Zhou P, Wu L, Wu K, Jiang W, Li J, Zhou L, Li X, Chang S, Huang Y, Tan H, Zhang G, He F, Wang Z: Overexpression of MMSET is correlation with poor prognosis in hepatocellular carcinoma. Pathol. Oncol. Res. 2013;19:303-309.

17 Sun H, Yu X, Wu W, Chen J, Shi M, Zheng L, Xu J: GLUT1 and ASCT2 as Predictors for Prognosis of Hepatocellular Carcinoma. PLoS ONE 2016;11:e0168907.

18 Sanjana NE, Shalem O, Zhang F: Improved vectors and genome-wide libraries for CRISPR screening. Nat Methods 2014;11:783-784.

19 Jiang W, Wang Z, Li X, Li J, Huang Y, Fan X, Duan Y: Reduced high-mobility group box 1 expression induced by RNA interference inhibits the bioactivity of hepatocellular carcinoma cell line HCCLM3. Dig Dis Sci 2012;57:92-98.

20 Li T, Fan J, Wang B, Traugh N, Chen Q, Liu JS, Li B, Liu XS: TIMER: A Web Server for Comprehensive Analysis of Tumor-Infiltrating Immune Cells. Cancer Res 2017;77:e108-e110.

21 Chung J, Park S, Park S, Park E, Cha P, Kim B, Chung Y, Woo S, Han C, Kim S, Suh K, Jang J, Lee K, Choi D, Lee S, Lee G, Hahm K, Shin J, Kim B, Noh K et al.: Overexpression of Romo1 promotes production of reactive oxygen species and invasiveness of hepatic tumor cells. Gastroenterology 2012;143:1084-1094.e1087.

-22 Hunecke D, Spanel R, Langer F, Nam SW, Borlak J: MYC-regulated genes involved in liver cell dysplasia identified in a transgenic model of liver cancer. J Pathol 2012;228:520-533.

-23 Zhang N, Wu Y, Lyu X, Li B, Yan X, Xiong H, Li X, Huang G, Zeng Y, Zhang Y, Lian J, Ni Z, He F: HSF1 upregulates ATG4B expression and enhances epirubicin-induced protective autophagy in hepatocellular carcinoma cells. Cancer Lett 2017;409:81-90.

24 Teng Y, Zhao H, Gao L, Zhang W, Shull A, Shay C: FGF19 Protects Hepatocellular Carcinoma Cells against Endoplasmic Reticulum Stress via Activation of FGFR4-GSK3 $\beta$-Nrf2 Signaling. Cancer Res. 2017;77:62156225.

25 Schleich S, Strassburger K, Janiesch P, Koledachkina T, Miller K, Haneke K, Cheng Y, Kuechler K, Stoecklin G, Duncan K, Teleman A: DENR-MCT-1 promotes translation re-initiation downstream of uORFs to control tissue growth. Nature 2014;512:208-212.

-26 Yan P, Gong H, Zhai X, Feng Y, Wu J, He S, Guo J, Wang X, Guo R, Xie J: Decreasing CNPY2 expression diminishes colorectal tumor growth and development through activation of p53 pathway. The American journal of pathology 2016;186:1015-1024.

27 Hong F, Liu B, Wu BX, Morreall J, Roth B, Davies C, Sun S, Diehl JA, Li Z: CNPY2 is a key initiator of the PERKCHOP pathway of the unfolded protein response. Nature Structural \& Molecular Biology 2017;24:834-839.

-28 Shi YH, Ding ZB, Zhou J, Hui B, Shi GM, Ke AW, Wang XY, Dai Z, Peng YF, Gu CY, Qiu SJ, Fan J: Targeting autophagy enhances sorafenib lethality for hepatocellular carcinoma via ER stress-related apoptosis. Autophagy 2011;7:1159-1172.

29 Sunami Y, Ringelhan M, Kokai E, Lu M, O'connor T, Lorentzen A, Weber A, Rodewald AK, Müllhaupt B, Terracciano L: Canonical NF - $\kappa$ B signaling in hepatocytes acts as a tumor - suppressor in hepatitis $B$ virus surface antigen - driven hepatocellular carcinoma by controlling the unfolded protein response. Hepatology 2016;63:1592-1607.

-30 Wang Y, Liu T, Tang W, Deng B, Chen Y, Zhu J, Shen X: Hepatocellular Carcinoma Cells Induce Regulatory T Cells and Lead to Poor Prognosis via Production of Transforming Growth Factor- $\beta 1$. Cell. Physiol. Biochem. 2016;38:306-318.

-31 Jin X, Moskophidis D, Mivechi N: Heat shock transcription factor 1 is a key determinant of HCC development by regulating hepatic steatosis and metabolic syndrome. Cell Metab. 2011;14:91-103.

32 Huang J, Qiu M, Wan L, Wang G, Huang T, Chen Z, Jiang S, Li X, Xie L, Cai L: TGF- $\beta 1$ Promotes Hepatocellular Carcinoma Invasion and Metastasis via ERK Pathway-Mediated FGFR4 Expression. Cell. Physiol. Biochem. 2018;45:1690-1699. 